\title{
Docetaxel for non-small-cell lung cancer harboring the activated EGFR mutation with T790M at initial presentation
}

This article was published in the following Dove Press journal:

OncoTargets and Therapy

5 March 2013

Number of times this article has been viewed

\section{Hiromichi Yamane' \\ Nobuaki Ochi' \\ Masayuki Yasugi ${ }^{2}$ \\ Takayuki Tabayashi' \\ Tomoko Yamagishi' \\ Yasumasa Monobe ${ }^{3}$ \\ Akiko Hisamoto ${ }^{4}$ \\ Katsuyuki Kiura ${ }^{4}$ \\ Nagio Takigawa'}

'Department of General Internal Medicine 4, Kawasaki Medical School, Okayama, Japan; ${ }^{2}$ Department of Respiratory Medicine, National Hospital Organization Fukuyama Medical Center, Fukuyama, Japan; ${ }^{3}$ Department of Pathology, Kawasaki Medical School Kawasaki Hospital, Okayama, Japan; ${ }^{4}$ Department of Hematology, Oncology, and Respiratory Medicine, Okayama University Graduate School of Medicine, Okayama, Japan
Correspondence: Hiromichi Yamane Department of General Internal Medicine 4, Kawasaki Medical

School, 2-I-80 Nakasange,

Okayama 700-8505, Japan

Tel +81 862252111

$\mathrm{Fax}+81862328343$

Email hiromichi.ya@gmail.com
Abstract: A 72-year-old woman was referred to our hospital with Stage IV non-small-cell lung cancer (NSCLC). Chest computed tomography revealed a mass in the upper lobe of the right lung, with pleural effusion. Cytologic examination identified adenocarcinoma cells in the right pleural effusion. Furthermore, both a deletion mutation in exon 19 and a threonine-methionine substitution mutation at position 790 in exon $20(\mathrm{~T} 790 \mathrm{M})$ were detected in the epidermal growth factor receptors (EGFR) in the malignant cells. As systemic chemotherapy consisting of carboplatin and pemetrexed or erlotinib proved ineffective, docetaxel monotherapy was initiated as a third-line treatment. Following salvage chemotherapy, her Eastern Cooperative Oncology Group performance status improved from 3 to 1 , with tumor regression over 5 months. To the best of our knowledge, this is the first report of successful docetaxel treatment for a patient with NSCLC harboring the T790M EGFR-activating mutation identified before treatment with EGFR tyrosine kinase inhibitors.

Keywords: non-small-cell lung cancer, EGFR mutation, pretreatment mutation, T790M, docetaxel

\section{Introduction}

Epidermal growth factor receptor (EGFR) gene mutation status is critical for predicting the clinical outcome of EGFR tyrosine kinase inhibitor (TKI) therapy in patients with non-small-cell lung cancer (NSCLC). Inhibition of EGFR activity improves treatment efficacy in patients with NSCLC harboring EGFR-activating mutations. EGFR-TKIs such as gefitinib or erlotinib attain a response rate of approximately $70 \%$ and progression-free survival of 9-13 months, although subgroups of patients experience long-lasting remission. ${ }^{1,2}$ Use of EGFR-TKIs as a first-line treatment in patients with advanced NSCLC harboring EGFR mutations improved progression-free survival with acceptable toxicity, compared with standard chemotherapy. EGFRTKIs are currently considered a standard first-line treatment for this disease entity. ${ }^{3-6}$ However, these tumors eventually develop resistance to EGFR-TKIs, possibly as a result of a secondary threonine-methionine substitution mutation at position 790 in exon 20 (T790M), MET amplification, or hepatocyte growth factor overexpression in relapsed tumors. ${ }^{7}$ At least half of the tumors that develop TKI resistance harbor a primary T790M EGFR mutation. ${ }^{7}$ However, multiple mechanisms underlie acquired EGFR-TKI resistance, and genetic heterogeneity of EGFR at each tumor site also contributes to the acquisition of drug resistance. ${ }^{8,9}$

At present, it is a standard practice in cases of acquired EGFR-TKI resistance to switch to conventional cytotoxic chemotherapy. ${ }^{7}$ An effective chemotherapeutic 
agent after acquisition of the T790M mutation and TKI resistance has yet to be established. In Japan, acquisition of the T790M mutation was reportedly associated with good clinical outcome. ${ }^{10}$ The reason for this result remains unclear; one explanation is that NSCLC cells that have acquired the T790M mutation show slower tumor growth than parental cells. ${ }^{11}$ Alternatively, some chemotherapeutic agents might have greater impact than others on this disease entity.

Differences have been reported between a T790M mutation that emerges after EGFR-TKI treatment and pretreatment T790M, suggesting that they are genetically distinct diseases. ${ }^{1}$ In the IRESSA Pan-Asia Study (IPASS) study, ${ }^{6}$ eleven treatment-naïve patients had an exon 20 T790M mutation: four had coexisting exon 19 deletions and three had coexisting L858R mutations. Three out of five patients with T790M mutations who received gefitinib attained a partial response (all three responders also had exon 19 deletions). ${ }^{6}$ Thus, EGFR-TKIs are suitable for NSCLC with a T790M mutation that coexists with activating mutations, such as exon 19 deletions or exon 21 point mutation. However, the effect of cytotoxic chemotherapy on NSCLC harboring both the pretreatment T790M and EGFR-activating mutations is unclear. ${ }^{12}$ We aimed to select an effective chemotherapeutic agent based on EGFR status, taking pre- or post-treatment T790M emergence into consideration. Here we present a case of successful treatment using single-agent docetaxel in a patient with NSCLC harboring both the pretreatment T790M and exon 19 deletion mutations.

\section{Case presentation}

A 72-year-old Japanese woman was referred to our hospital for treatment of NSCLC. One month earlier, she consulted another clinic with an intractable cough, hematemesis, and $10 \%$ body weight loss in the preceding 6 months. Her Eastern Cooperative Oncology Group performance status (PS) was 1. She had no history of smoking. Chest computed tomography (CT) revealed a pulmonary mass in the upper lobe of the right lung, with pleural indentation and massive pleural effusion (Figure 1A). Magnetic resonance imaging of the brain detected no evidence of metastasis, but a metastatic liver tumor was detected by abdominal CT (Figure 1B). Levels of the serum tumor markers carcinoembryonic antigen (CEA) and carbohydrate antigen 19-9 (CA19-9) were elevated (CEA: $11 \mathrm{ng} / \mathrm{mL}$, reference value: $<5 \mathrm{ng} / \mathrm{mL}$; CA19-9: $1747 \mathrm{ng} / \mathrm{mL}$, reference value: $<39 \mathrm{ng} / \mathrm{mL}$ ). Bronchoscopic and cytologic examination of the right pleural effusion revealed poorly differentiated adenocarcinoma (Figure 2A). The patient was diagnosed with advanced NSCLC (T2aN2M1b, Stage IV). The T790M EGFR mutation in exon 20 and an in-frame deletion in exon 19 were detected in tumor cells from the right pleural effusion using the peptide nucleic acid-locked nucleic acid polymerase chain reaction clamp (PNA-LNA PCR clamp) method (Figure 2B).

The patient received systemic chemotherapy consisting of carboplatin and pemetrexed. Chest CT after three cycles of chemotherapy showed disease progression with a PS of 2. Subsequently, she was treated with erlotinib. However, she complained of chest and abdominal pain and her PS deteriorated to 3 during the second-line treatment. Then, we examined her using a chest $\mathrm{CT}$ at day 14 of the treatment of EGFR-TKI. The chest CT scan at day 14 after EGFR-TKI initiation revealed multiple liver metastases, increased right pleural effusion,
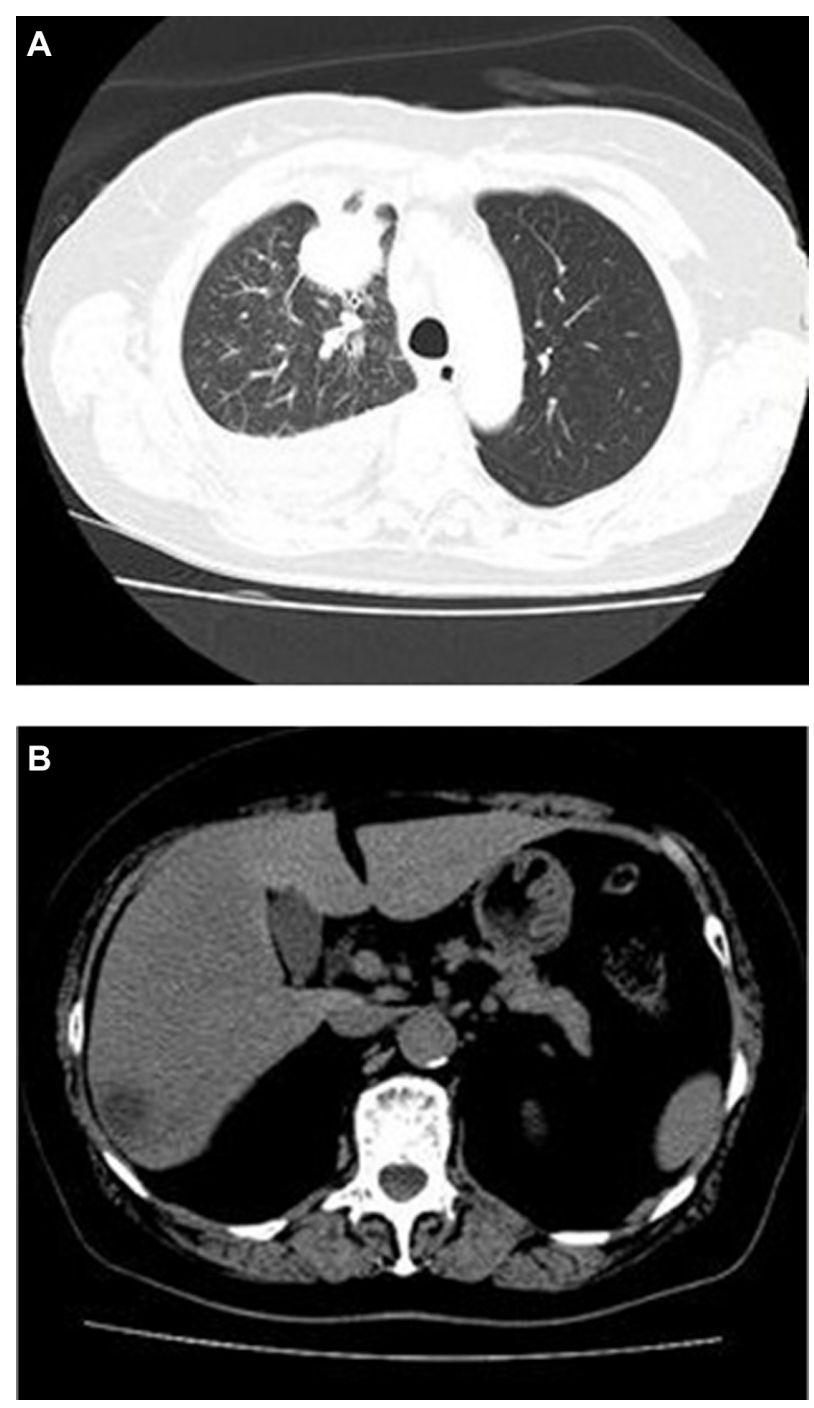

Figure I (A) Chest CT revealed a mass in the right upper lobe, with pleural effusion. (B) Abdominal CT revealed a low-density lesion in the liver. Abbreviation: CT, computed tomography. 


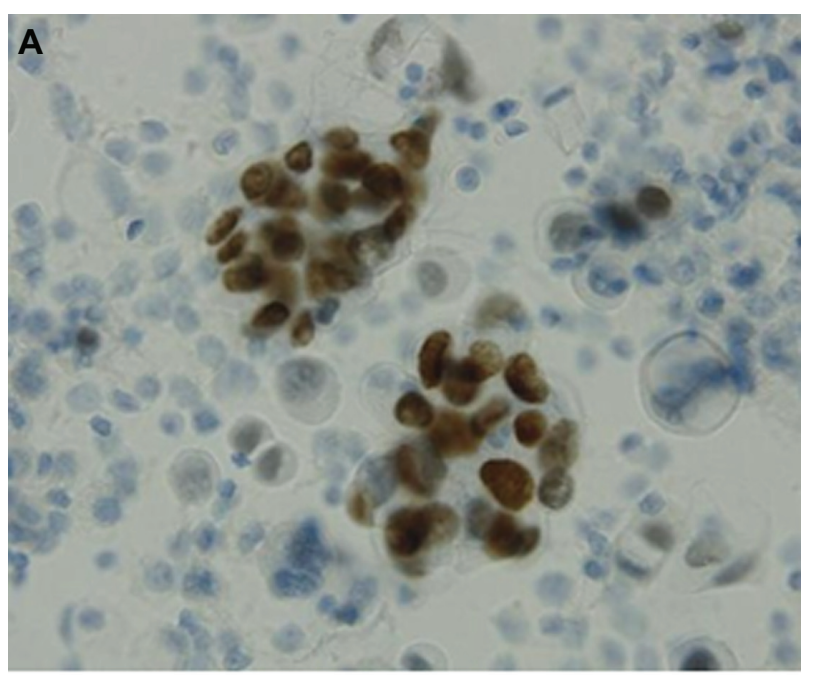

B
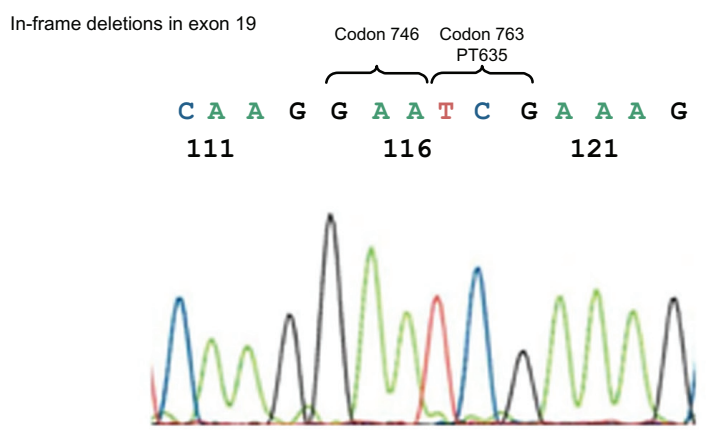

Mutation at T790M in exon 20
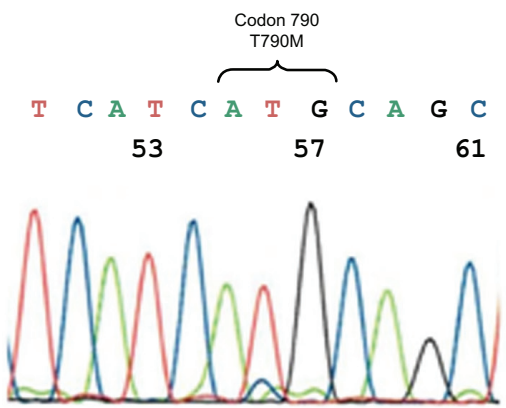

Figure 2 (A) Adenocarcinoma cells were evident in the right pleural effusion. TTF-I was detected on tumor cell surfaces by immunohistochemical staining. (B) Tumor cells from the right pleural effusion harbored both a pretreatment T790M EGFR mutation in exon 20 and in-frame deletions in exon 19.

Note: This result was obtained using the PNA-LNA PCR clamp method. Abbreviations: EGFR, epidermal growth factor receptor; PNA-LNA PCR, peptide nucleic acid-locked nucleic acid polymerase chain reaction; T790M, threoninemethionine substitution mutation at position 790 in exon 20; TTF-I, thyroid transcription factor-I.

enlargement of multiple metastatic liver tumors, and atelectasis of the middle lobe (Figures 3A and S1).

Although her PS deteriorated to 3, she wished to undergo further chemotherapy. Docetaxel monotherapy was initiated as salvage chemotherapy. A chest CT after two and four cycles of this treatment (Figure 3B and C) revealed tumor regression and decreased right pleural effusion.
No hematologic or nonhematologic toxicities of Grade 2 or higher were observed. Her disease continued to be stable, with an improved PS of 1, for 5 months, until multiple metastatic brain tumors were detected. Her PS deteriorated to 3, and finally, she decided against receiving further chemotherapy. Two months after chemotherapy discontinuation, she died as a result of multiple brain metastases, which progressed despite brain irradiation.

\section{Discussion}

In the survival analyses of two randomized Phase III studies, ${ }^{13,14}$ there was no statistically significant difference between gefitinib and docetaxel as salvage treatments for NSCLC with EGFR-activating mutations. Docetaxel significantly improved progression-free survival and response rate compared with erlotinib in patients with wild-type EGFR. ${ }^{15}$ Meanwhile, patients with NSCLC harboring the Kirsten rat sarcoma viral oncogene homolog mutation did not respond to docetaxel monotherapy. ${ }^{16}$ This suggests that effective chemotherapeutic drugs for NSCLC should be sought according to the mutation status of driver oncogenes. It has been recently demonstrated in vitro ${ }^{17}$ that NSCLC cell lines that acquired EGFR-TKI resistance were also resistant to chemotherapeutic drugs. However, the efficacy of anticancer agents for such resistant tumors has not been investigated by clinical trials. Hence, we considered it important to report the effectiveness of chemotherapeutic drugs in a patient with NSCLC harboring the pretreatment T790M and EGFRactivating mutations.

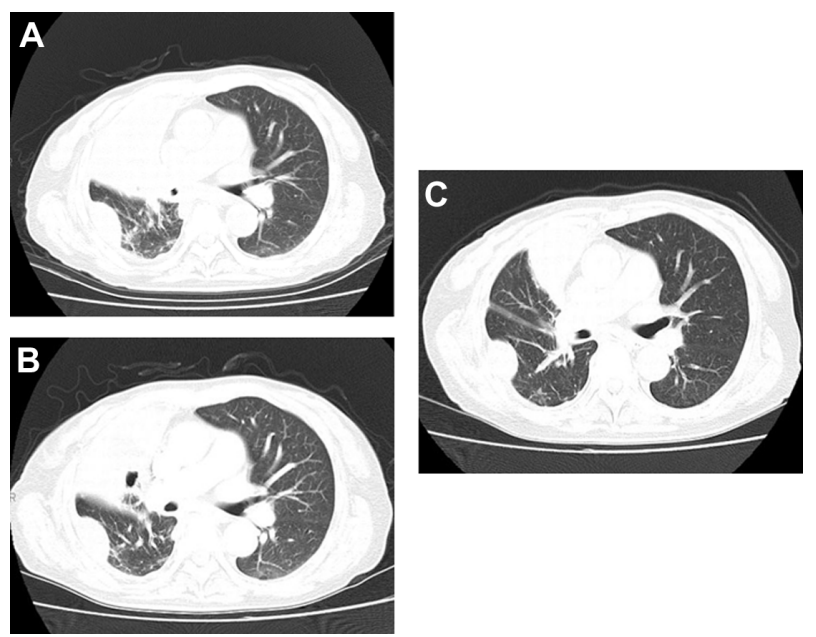

Figure 3 (A) Chest CT before docetaxel monotherapy revealed atelectasis of the right middle lobe, with right pleural effusion. (B) Chest CT revealing tumor response after two cycles of docetaxel. (C) Chest CT revealing tumor response after four cycles of docetaxel.

Abbreviation: CT, computed tomography. 
The pretreatment T790M mutation is considered to be present in tumor cells before EGFR-TKI treatment in a subpopulation of individuals. Historically, pretreatment T790M mutation-positive patients were detected by widely used and highly sensitive methods such as the PNA-LNA PCR clamp or Scorpion Amplified Refractory Mutation System methods, with an analytic sensitivity of approximately $0.1 \%-1.0 \%{ }^{12}$ These patients demonstrated a shorter EGFRTKI response duration. ${ }^{1,9,18,19}$ However, pretreatment T790M mutation analysis by Fujita et a $1^{12}$ revealed a prolonged EGFR-TKI response duration in patients with NSCLC harboring an EGFR-activating mutation. They also showed that the pretreatment $\mathrm{T} 790 \mathrm{M}$ mutation could be detected at a frequency of $79 \%$, using a supersensitive, modified colony hybridization $(\mathrm{CH})$ technique with an analytic sensitivity of approximately $0.01 \%$. In their report, ${ }^{12}$ patients with strong positivity reported a significantly longer time to treatment failure compared with those with modest positivity or lacking the T790M mutation. Detection of patients harboring the pretreatment T790M mutation has greatly improved with the development of the modified $\mathrm{CH}$ technique. A prospective clinical study that investigates the efficacy of docetaxel in treating this disease entity is now warranted. However, the $\mathrm{CH}$ technique is not considered an optimal method to detect EGFR mutations in clinical settings. Thus, based both on previous clinical reports ${ }^{1,9,18,19}$ and our experience of this case, through widely used methods, we inferred that the pretreatment T790M mutation was associated with shorter progression-free survival after EGFR-TKI therapy.

Since 2000, several large-scale clinical trials have been conducted to determine the standard chemotherapy regimen for the second-line treatment of advanced NSCLC that has relapsed or progressed after platinum-based chemotherapy. ${ }^{20-22}$ Docetaxel monotherapy was the first US Food and Drug Administration-approved chemotherapy regimen for this disease entity. ${ }^{20,21}$ Furthermore, both pemetrexed and docetaxel were recommended as standard second-line treatments for advanced NSCLC since 2004. ${ }^{22}$ However, from the perspective of recent developments in molecular classification, we suggest an investigation of the efficacy of docetaxel and pemetrexed for NSCLC according to the EGFR mutation status in prospective clinical trials.

A recent report by Bai et al ${ }^{23}$ provides evidence that first-line chemotherapy may influence EGFR mutation status in both tissue and peripheral blood samples. In their report, a decrease in EGFR mutation rate was significantly associated with better clinical response to chemotherapeutic agents. This result indicates that a biomarker of therapy resistance should be investigated before switching to another treatment. Rebiopsy of the relapsed tumor specimen or biomarker analysis using a peripheral blood sample are some of the concrete methods that can be used to make a reasonable treatment decision in the future.

Although it may be difficult to make a treatment decision in several situations, we make the following recommendations. If an EGFR-activating mutation is discovered during first-line chemotherapy, a switch to EGFR-TKI is reasonable. However, if first-line chemotherapy proves to be effective, it should be continued until disease progression and then EGFR-TKI will be useful as salvage treatment. If resistance to EGFR-TKI for lung cancer which harbored an EGFR-activating mutation is clinically suspected, an EGFRTKI-resistant mutation such as T790M should be investigated and then standard chemotherapy should be considered.

\section{Conclusion}

During the clinical course of treatment for our patient, docetaxel was effective, contrary to our pretreatment expectations. To the best of our knowledge, our case is the first clinical report describing the efficacy of docetaxel monotherapy for NSCLC harboring both the T790M EGFR mutation in exon 20 and another EGFR-activating mutation. We think that further demonstrations of the results of this case report are necessary in a larger patient cohort. In addition, we understand that more data are needed before this strategy can be widely accepted. We expect that new and targeted agents for the treatment of patients such as in our case will be available in the near future.

\section{Author contributions}

Hiromichi Yamane, Nobuaki Ochi, Yasumasa Monobe, and Masayuki Yasugi analyzed the bibliographical data. Hiromichi Yamane, Masayuki Yasugi, Nobuaki Ochi, Takayuki Tabayashi, Tomoko Yamagishi, Akiko Hisamoto, Katsuyuki Kiura, and Nagio Takigawa were involved in the case management of the patient. Hiromichi Yamane and Nagio Takigawa wrote the paper.

\section{Acknowledgments}

The authors would like to thank Enago (http://www.enago.jp; Mumbai, India) for the English language review.

\section{Disclosure}

Drs Katsuyuki Kiura and Nagio Takigawa have received honoraria from Chugai Pharmaceutical and Sanofi-Aventis KK. The authors report no other conflicts of interest in this work. 


\section{References}

1. Su KY, Chen HY, Li KC, et al. Pretreatment epidermal growth factor receptor (EGFR) T790M mutation predicts shorter EGFR tyrosine kinase inhibitor response duration in patients with non-small-cell lung cancer. J Clin Oncol. 2012;30(4):433-440.

2. Mayo C, Bertran-Alamillo J, Molina-Vila MÁ, Giménez-Capitán A, Costa C, Rosell R. Pharmacogenetics of EGFR in lung cancer: perspectives and clinical applications. Pharmacogenomics. 2012; 13(7):789-802.

3. Maemondo M, Inoue A, Kobayashi K, et al; North-East Japan Study Group. Gefitinib or chemotherapy for non-small-cell lung cancer with mutated EGFR. N Engl J Med. 2010;362(25):2380-2388.

4. Mok TS, Wu YL, Thongprasert S, et al. Gefitinib or carboplatin paclitaxel in pulmonary adenocarcinoma. $N$ Engl J Med. 2009; 361(10):947-957.

5. Mitsudomi T, Morita S, Yatabe Y, et al; West Japan Oncology Group. Gefitinib versus cisplatin plus docetaxel in patients with non-smallcell lung cancer harbouring mutations of the epidermal growth factor receptor (WJTOG3405): an open label, randomised phase 3 trial. Lancet Oncol. 2010:11(2):121-128.

6. Fukuoka M, Wu YL, Thongprasert S, et al. Biomarker analyses and final overall survival results from a phase III, randomized, open-label, first-line study of gefitinib versus carboplatin/paclitaxel in clinically selected patients with advanced non-small-cell lung cancer in Asia (IPASS). J Clin Oncol. 2011;29(21):2866-2874.

7. Oxnard GR, Arcila ME, Chmielecki J, Ladanyi M, Miller VA, Pao W. New strategies in overcoming acquired resistance to epidermal growth factor receptor tyrosine kinase inhibitors in lung cancer. Clin Cancer Res. 2011;17(17):5530-5537.

8. Jiang SX, Yamashita K, Yamamoto M, et al. EGFR genetic heterogeneity of non-small-cell lung cancers contributing to acquired gefitinib resistance. Int J Cancer. 2008;123(11):2480-2486.

9. Rosell R, Molina MA, Costa C, et al. Pretreatment EGFR T790M mutation and BRCA1 mRNA expression in erlotinib-treated advanced non-small-cell lung cancer patients with EGFR mutations. Clin Cancer Res. 2011;17(5):1160-1168.

10. Uramoto H, Yano S, Tanaka F. T790M is associated with a favorable prognosis in Japanese patients treated with an EGFR-TKI. Lung Cancer. 2012;76(1):129-130.

11. Chmielecki J, Foo J, Oxnard GR, et al. Optimization of dosing for EGFR-mutant non-small cell lung cancer with evolutionary cancer modeling. Sci Transl Med. 2011;3(90):90ra59.

12. Fujita Y, Suda K, Kimura H, et al. Highly sensitive detection of EGFR T790M mutation using colony hybridization predicts favorable prognosis of patients with lung cancer harboring activating EGFR mutation. J Thorac Oncol. 2012;7(11):1640-1644.
13. Douillard JY, Shepherd FA, Hirsh V, et al. Molecular predictors of outcome with gefitinib and docetaxel in previously treated non-smallcell lung cancer: data from the randomized phase III INTEREST trial. J Clin Oncol. 2010;28(5):744-752.

14. Maruyama R, Nishiwaki Y, Tamura T, et al. Phase III study, V-15-32, of gefitinib versus docetaxel in previously treated Japanese patients with non-small-cell lung cancer. J Clin Oncol. 2008;26(26):4244-4252.

15. Garassino MC, Martelli O, Bettini A, et al. TAILOR: A phase III trial comparing erlotinib with docetaxel as the second-line treatment of NSCLC patients with wild-type (wt) EGFR. Proceedings of the 48th ASCO Annual Meeting; June 1-5, 2012; Chicago, USA.

16. Jänne PA, Shaw AT, Pereira JR, et al. Selumetinib plus docetaxel for KRASmutant advanced non-small-cell lung cancer: a randomised, multicentre, placebo-controlled, phase 2 study. Lancet Oncol. 2013;14(1):38-47.

17. Ercan D, Xu C, Yanagita M, et al. Reactivation of ERK Signaling Causes Resistance to EGFR Kinase Inhibitors. Cancer Discov. 2012; 2(10):934-947.

18. Maheswaran S, Sequist LV, Nagrath S, et al. Detection of mutations in EGFR in circulating lung-cancer cells. $N$ Engl J Med. 2008; 359(4):366-377.

19. Mack PC, Holland WS, Burich RA, et al. EGFR mutations detected in plasma are associated with patient outcomes in erlotinib plus docetaxel-treated non-small cell lung cancer. J Thorac Oncol. 2009; 4(12):1466-1472.

20. Shepherd FA, Dancey J, Ramlau R, et al. Prospective randomized trial of docetaxel versus best supportive care in patients with non-small-cell lung cancer previously treated with platinum-based chemotherapy. J Clin Oncol. 2000;18(10):2095-2103.

21. Fossella FV, DeVore R, Kerr RN, et al. Randomized phase III trial of docetaxel versus vinorelbine or ifosfamide in patients with advanced non-small-cell lung cancer previously treated with platinum-containing chemotherapy regimens. The TAX 320 Non-Small Cell Lung Cancer Study Group. J Clin Oncol. 2000;18(12):2354-2362.

22. Hanna N, Shepherd FA, Fossella FV, et al. Randomized phase III trial of pemetrexed versus docetaxel in patients with non-small-cell lung cancer previously treated with chemotherapy. J Clin Oncol. 2004;22(9):1589-1597.

23. Bai H, Wang Z, Chen $\mathrm{K}$, et al. Influence of chemotherapy on EGFR mutation status among patients with non-small-cell lung cancer. J Clin Oncol. 2012;30(25):3077-3083. 


\section{Supplementary materials}
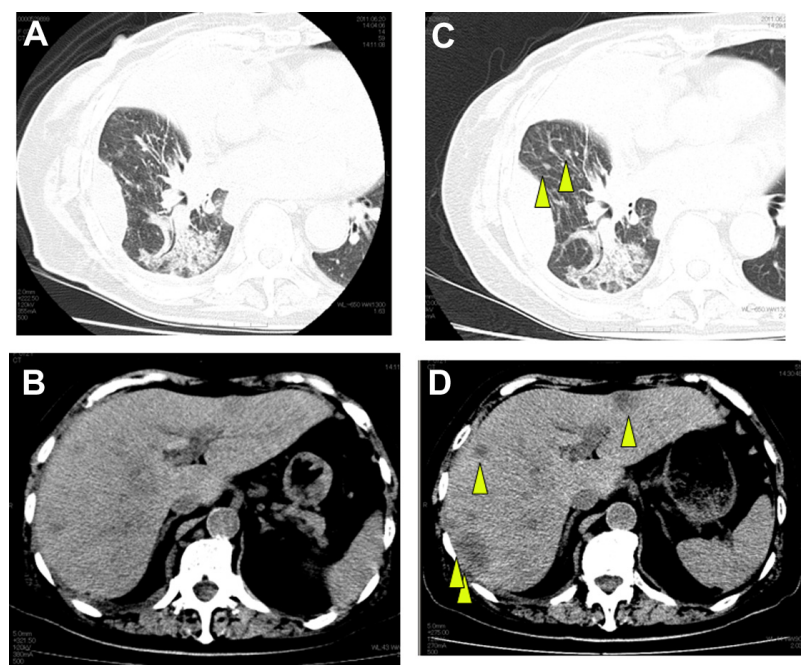

Figure SI (A) Chest CT just before erlotinib monotherapy. (B) Abdominal CT just before erlotinib monotherapy revealed multiple liver metastases. (C) Chest CT at day 14 of erlotinib monotherapy. Pulmonary small nodules of right lower lobe appeared (arrowhead). (D) Abdominal CT at day I4 of erlotinib monotherapy revealed enlarged liver metastases (arrowhead).

Abbreviation: CT, computed tomography.

\section{Publish your work in this journal}

OncoTargets and Therapy is an international, peer-reviewed, open access journal focusing on the pathological basis of all cancers, potential targets for therapy and treatment protocols employed to improve the management of cancer patients. The journal also focuses on the impact of management programs and new therapeutic agents and protocols on patient perspectives such as quality of life, adherence and satisfaction. The manuscript management system is completely online and includes a very quick and fair peer-review system, which is all easy to use. Visit http://www.dovepress.com/testimonials.php to read real quotes from published authors. 